\title{
Clinical characterization of patients with non- small cell lung cancer according to the intensity of smoking and stage in which non-small cell lung cancer is diagnosed
}

\begin{abstract}
The high incidence and mortality recorded in lung cancer make it a worrying health problem worldwide and nationally. Although the relationship between smoking and this type of neoplasm is unquestionable, it does not seem to be a simple association of cause and effect. In order to characterize patients with non-small cell lung cancer according to the intensity of smoking and the stage of the disease at the time of diagnosis, a descriptive, cross-sectional, retrospective study was carried out in a sample of 55 patients with this disease.
\end{abstract}

Result: $85,7 \%$ of the women were diagnosed in stage IV. Among patients over 75 years of age, about $50 \%$ were diagnosed with stage II. More than $95 \%$ of the women with a history of COPD were in the most advanced stage. $80 \%$ of those who smoked for more than 60 years were diagnosed in stage II; while $60 \%$ of those who had more than 10 years of abstinence were also located in this stage.

Conclusion: The present study suggests that the female gender, history of COPD, and an age of early initiation smoking, are predominant clinical features of patients with NSCLC detected in more advanced stages of the disease cancer. The relationship between age, the number of cigarettes smoked per day, or time smoking with the severity of the disease is not clear; It is very likely that other factors are involved.

Keywords: non-small cell lung cancer, theory of adaptive oncogenesis and pulmonary carcinogenesis, theory of somatic mutation and pulmonary carcinogenesis
Volume 5 Issue 2 - 2018

\section{Armando Ernesto Pérez Cala,' Edgar Benítez Sánchez, ${ }^{2}$ Ernesto Ramirez Ramirez, ${ }^{3}$ Dennis Sánchez Ramos, ${ }^{3}$ Yamile Hinojosa Rivera' \\ 'First Degree Specialist in Internal Medicine,Assistant Professor of Internal Medicine, Auxiliary Researcher, National Institute of Endocrinology, Cuba \\ ${ }^{2}$ First Degree Specialist in Hygiene and Epidemiology, Instructor Professor of Hygiene and Epidemiology, Master in Infectious Diseases, National Institute of Endocrinology, Cuba ${ }^{3}$ First Degree Specialist in Internal Medicine, National Institute of Endocrinology, Cuba}

Correspondence: Armando Ernesto Pérez Cala, First Degree Specialist in Internal Medicine, Assistant Professor of Internal Medicine, Auxiliary Researcher, Master in Medical Emergencies, Cuba, Email armando.perez@infomed.sld.cu

Received: March 03, 2017 | Published: March 16, 2018

\section{Introduction}

Neoplastic diseases are a serious health problem worldwide, and their incidence is increasing. Currently about 14 million new cases are registered annually; it is estimated that by 2030 the reported figures of people diagnosed with some type of malignant tumour are close to 30 million per year. ${ }^{1-4}$ There is also a sad contrast in terms of mortality from malignant diseases between developed countries and those in the process of development. While in the former the tendencies point towards a reduction of the mortality, in the underdeveloped countries this health indicator is increasing; this fact, together with the proportional rise in incidence, adds to the developing nations in what is known as: double burden of disease due to cancer.,

Among all varieties of malignant tumours, lung cancer tops the list both in cancer incidence and mortality in men, and is the fourth most frequent neoplasia in women, although the second cause of death from neoplastic diseases in people belonging to this sex. ${ }^{1}$ It is alarming that the incidence of this tumour in female patients has shown a substantial increase in recent decades. ${ }^{1}$ Cuba shares with the USA the same rates of incidence and mortality from lung cancer; and it is significant that the "female phenomenon" behaves in the same way. ${ }^{3}$ During the past year, in our country there were 3482 deaths from lung cancer in men (rate adjusted for sex of 62,2 per 100 thousand inhabitants), and 2053 deaths in women due to the same cause (rate adjusted for sex of 36,4 per 100 thousand inhabitants). ${ }^{5}$

Much has been insisted, and demonstrated, on the strong association between smoking and the risk of developing lung cancer. In fact, there is no doubt that many of the components of the combustion of carcinogenic effect snuff have ${ }^{6,7}$ However, this causeeffect relationship is still complex and influenced by various factors, say biological, behavioral, genetic, social, gender, and even ethnic. ${ }^{8-10}$ Hence, the diversity of predictive models that compete for degrees of validity and effectiveness. ${ }^{11,12}$

Currently, cancer, and the process from which it develops, carcinogenesis, is defined as a variety of somatic evolution, which compiles exactly with the most elementary and universal Darwinian laws. ${ }^{13}$

Evolutionary dynamics in which deterministic and random forces intervene, imbricated in a complex ecological framework. Biological and evolutionary investigations of cancer have led to promising results; and contradictions. Two paradigms of carcinogenesis from an ecological-evolutionary perspective have been raised in the last decades: The theory of somatic mutation and the theory of adaptive oncogenesis. The first, predominant, proposes that a tumour develops as sequential and progressive accumulation of mutations that increase 
the degree conductive adaptive fitness of mutated cells until they take all the phenotypic characteristics of malignant cells ${ }^{14,15}$ occurs. The theory of adaptive oncogenesis in turn suggests that the transforming nature of oncogenic mutations depends tissue context in which they occur, and not the simple accumulation in time and space. ${ }^{16,17}$ The practical implications of both paradigms are still evident, to the point that there are authors who, based on the theory of somatic mutation, have advocated that two thirds of all human tumours are caused by the accumulation of mutations during normal divisions of the stem cells contained in the tissues. ${ }^{18}$

Lung cancer is not excluded from the debate, since from the previous assertions, it has been suggested that the effect of smoking on the risk of developing this neoplasm is when more "additive". ${ }^{18}$ Then, then, who smokes and develops a lung cancer, they simply have "bad luck"? Is smoking limited to increasing the risk of accumulation of oncogenic mutations, and therefore, those individuals "favored" by chance, or their genetics, can smoke?

Apart from the causal effect of smoking on the risk of lung cancer, little has been said about the influence of this harmful habit on the severity of the disease at the time of diagnosis; or raised in the form of a question: is there any relationship between the different variables of tobacco dependence (intensity and duration of habit, sex, association with other diseases, etc.) and the extent of the disease once diagnosed? In order to satisfy this question, the authors decided to carry out this work.

\section{Objective}

Clinically characterize patients with non-small cell lung cancer according to the intensity of smoking and the stage of the disease at the time of diagnosis.

\section{Materials and methods}

A descriptive, transversal, retrospective study was carried out. The universe consisted of patients admitted to the military hospital Dr. Joaquín Castillo Duany with a diagnosis of lung cancer during the period between January 2009 and January 2015. Out of a total of 112 patients, a sample of 55 individuals was selected by the random probabilistic method. The inclusion criteria were taken into account: the histological confirmation of the neoplasm, its membership in the group of non-small cell lung tumours; as well as the histological subcategory corresponding to this group (adenocarcinoma, squamous cell carcinoma, giant cell carcinoma, etc.); performing computed tomography (CT) and/or single thoracic chest radiograph each of the participants of the investigation. The degree of histological differentiation of the neoplasm's was not taken into account because it was not a constant element in the cytological reports of sputum or pleural fluid, the most frequent source, in this study, of histological confirmation. The clinical-demographic variables to be considered were age (less than 60 years, between 61 and 75 years, and older than 75 years); and sex (male or female). Variables expressing the intensity of smoking were considered: age of initiation in smoking habit (less than 15 years, between 15 and 20 years, and above 20 years); number of cigarettes per day (less than 20, between 20 and 40, or more than 40 cigarettes per day); ex-smoker's condition and time of abstinence (less than 5 years, between 5 and 10 years, and more than 10 years); time smoking at the time of diagnosis (from 30 to 39 years, between 40 to 49 years, between 50 to 59 years, and more than 60 years); presence of a history of chronic obstructive disease (COPD). The stage of the tumour at diagnosis (I, II, III, and IV) was used as a variable that expressed the severity of the disease.

For the consideration of the history of COPD, and given the difficulties in terms of its diagnosis and classification still existing; together with its close link to lung cancer, the operative criteria of the American Thoracic Society (TAS) were taken into account: previous history of admissions with this diagnosis, outpatient follow-up for this cause, and the elements clinical, humoral, and imaging findings suggesting bronchial obstruction/air entrapment (except hypocrotic fingers, polyglobulia, and elevated hematocrit when compared with lung cancer). ${ }^{19}$

Regarding the stage of the disease at the time of diagnosis, and in order to make it simpler, we did not consider the complete TNM classification system traditionally used, but the tomographic and/ or radiographic characteristics that define size, location, tumour extension, and lymph node involvement. In this way, the stages were defined as: stage I: tumor smaller than 3 centimeters $(\mathrm{cms})$, without invasion more proximal than the lobar bronchus; stage II: tumor greater than $3 \mathrm{~cm}$, which affects the main bronchus more than $2 \mathrm{~cm}$ from the carina, invades the visceral pleura, or produces atelectasis or obstructive pneumonitis to the hilar region; stage III: tumor of any size with invasion of the thoracic wall, diaphragm, mediastinal pleura or pericardium, or that affects the main bronchus less than 2 $\mathrm{cm}$ from the carina, or produces atelectasis or obstructive pneumonitis of the entire lung; and stage IV: tumor of any size that invades the mediastinum, heart, large vessels, trachea, oesophagus, vertebral body, carina, causes pleural or pericardial effusion, or is accompanied by satellite lymph nodes on the same side of the primary tumor. ${ }^{20}$

Summary measures were used for the qualitative variables: the absolute and relative frequency and the average for the quantitative ones. All data were obtained from the review of medical records.

\section{Results}

The association between the gender and stage of diagnosis of lung cancer is of great interest. In the present investigation, more than $20 \%$ of male patients were diagnosed in stage II of the disease. In regard to women, $85,7 \%$ was diagnosed in stage IV, while the remaining percentage was detected in stage III. There was no registry of female patients diagnosed in early stages of this neoplasm (Table 1).

Age was another significant aspect of the study. All patients under 60 years of age were diagnosed at the most advanced stage of the neoplastic pulmonary process. In the age group between 61 and 75 years, more than $70 \%$ of the patients were in stage IV of the disease at the time of diagnosis. It is striking that among patients over 75 years of age, close to $50 \%$ were diagnosed in stage II (Table 1).

Regarding the histological type, 40 patients were carriers of adenocarcinoma as a histological variety of lung cancer $(72,7 \%)$. Of these, $77.5 \%$ was detected in the most advanced stage. Although the individuals affected by the epidermoid variety represented a minority in the sample (15 cases), about $30 \%$ were in stage II of the disease (Table 1).

The history of COPD and sex was taken into account. In a very contrasting way, more than $95 \%$ of the women with COPD were diagnosed in the stage IV of the nosological process, while $75 \%$ of the men affected by this pathology were found to carry a malignant neoplasm of the lung in the most advanced stage; with $10 \%$ detection in stages I and II, respectively, in this same sex (Table 1). 
Table I Distribution of patients according to sex, age, histological type, history of COPD and tumor stages

\begin{tabular}{|c|c|c|c|c|c|c|c|c|c|}
\hline \multirow{3}{*}{ Variables } & \multicolumn{9}{|c|}{ Stages at diagnosis } \\
\hline & \multicolumn{2}{|l|}{$\mathbf{I}$} & \multicolumn{2}{|l|}{ II } & \multicolumn{2}{|l|}{ III } & \multicolumn{2}{|l|}{ IV } & \multirow{2}{*}{$\begin{array}{l}\text { Total } \\
\text { Do not. }\end{array}$} \\
\hline & Do not. & $\%$ & Do not. & $\%$ & Do not. & $\%$ & Do not. & $\%$ & \\
\hline \multicolumn{10}{|l|}{ Sex } \\
\hline Male & 2 & 4.9 & 9 & 22 & 3 & 7.3 & 27 & 65.9 & 41 \\
\hline Female & 0 & 0 & 0 & 0 & 2 & 14.3 & 12 & 85.7 & 14 \\
\hline \multicolumn{10}{|c|}{ Age groups (years) } \\
\hline Minus 60 & 0 & 0 & 0 & 0 & 0 & 0 & 4 & 100 & 4 \\
\hline 60 to 75 & 1 & 2.9 & I & 2.9 & 5 & 14.7 & 27 & 79.4 & 3.4 \\
\hline More 75 & 1 & 5.9 & 8 & 47.1 & 0 & 0 & 8 & 47.1 & 17 \\
\hline \multicolumn{10}{|l|}{ *APP COPD } \\
\hline Male & 2 & 10 & 2 & 10 & 1 & 5 & fifteen & 75 & twenty \\
\hline Female & 0 & 0 & 0 & 0 & 1 & 7.7 & 12 & 92.3 & 13 \\
\hline \multicolumn{10}{|c|}{ Histological type } \\
\hline Adenocarcinoma & 1 & 2.5 & 5 & 12.5 & 3 & 7.5 & 31 & 77.5 & 40 \\
\hline Epidermoid & 1 & 6.7 & 4 & 26.7 & 2 & 13.3 & 8 & 53.3 & fifteen \\
\hline
\end{tabular}

*APP, Personal pathological background; Sources, Data obtained from the medical records

The age of initiation was also evaluated: In the group of patients whose history of smoking dates from a range of age less than 15 years, $8,3 \%$ of these were represented in stages I, II and III of the disease. However, $75 \%$ were located in stage IV at the time of diagnosis. Already in the group of those who started between 15 and 20 years, the percentages of detection in the earliest stages showed a discreet but obvious increase (11, 7\% in stage II); as well as a decrease of
$5 \%$ in terms of stage IV detection. Although most of the individuals participating in this study had started smoking at an age older than 20 years, more than $20 \%$ of these were located in stage II of the tumor at the time of detection, at the same time it showed the lowest percentage $(69,2 \%)$ of patients detected in stage IV compared to the groups of patients that started at younger ages (Table 2).

Table 2 Distribution of patients according to variables of smoking intensity and tumor stage

\begin{tabular}{|c|c|c|c|c|c|c|c|c|c|}
\hline \multirow{3}{*}{ Variables } & \multicolumn{9}{|c|}{ Stages at diagnosis } \\
\hline & \multicolumn{2}{|l|}{$\mathbf{I}$} & \multicolumn{2}{|l|}{ II } & \multicolumn{2}{|l|}{ III } & \multicolumn{2}{|l|}{ IV } & \multirow{2}{*}{$\begin{array}{l}\text { Total } \\
\text { Do not. }\end{array}$} \\
\hline & Do not. & $\%$ & Do not. & $\%$ & Do not. & $\%$ & Do not. & $\%$ & \\
\hline \multicolumn{10}{|c|}{ Age of initiation of smoking } \\
\hline Less than 15 & 1 & 8.3 & 1 & 8.3 & 1 & 8.3 & 9 & 75 & 12 \\
\hline 15 to 19 & 0 & 0 & 2 & 11.8 & 3 & 17.6 & 12 & 70.6 & 17 \\
\hline 20 and more & 1 & 3.8 & 6 & 23.1 & 1 & 3.8 & 18 & 69.2 & 26 \\
\hline \multicolumn{10}{|l|}{ Cigars $x$ day } \\
\hline Less than 20 & 0 & 0 & 0 & 0 & 0 & 0 & 0 & 0 & 0 \\
\hline
\end{tabular}


Table continued....

\begin{tabular}{|c|c|c|c|c|c|c|c|c|c|}
\hline \multirow{4}{*}{ Variables } & \multicolumn{9}{|c|}{ Stages at diagnosis } \\
\hline & & & & & & & & & \\
\hline & \multicolumn{2}{|l|}{$\mathbf{I}$} & \multicolumn{2}{|l|}{ II } & \multicolumn{2}{|l|}{ III } & \multicolumn{2}{|l|}{ IV } & \multirow{2}{*}{$\begin{array}{l}\text { Total } \\
\text { Do not. }\end{array}$} \\
\hline & Do not. & $\%$ & Do not. & $\%$ & Do not. & $\%$ & Do not. & $\%$ & \\
\hline Between 20 and 40 & 2 & 4.3 & 7 & 14.9 & 5 & 10.6 & 33 & 70.2 & 47 \\
\hline More than 40 & 0 & 0 & 2 & 25 & 0 & 0 & 6 & 75 & 8 \\
\hline \multicolumn{10}{|c|}{ Smoking time (years) } \\
\hline 30 to 39 & 0 & 0 & 0 & 0 & 1 & 20 & 4 & 80 & 5 \\
\hline 40 to 49 & 0 & 0 & 0 & 0 & 2 & 10.5 & 17 & 89.5 & 19 \\
\hline 50 to 59 & 2 & 8.3 & 5 & 20.8 & 1 & 4.2 & 16 & 66.7 & 24 \\
\hline 60 and more & 0 & 0 & 4 & 40 & 1 & 10 & 5 & 50 & 10 \\
\hline Former smoker & 2 & 11.8 & 9 & 52.9 & 1 & 5.9 & 5 & 29.4 & 17 \\
\hline \multicolumn{10}{|c|}{ Ex-smoker's time (years) } \\
\hline Less than 5 & 0 & 0 & 0 & 0 & 0 & 0 & 0 & 0 & 0 \\
\hline 5 to 10 & 2 & 22.2 & 4 & 44.4 & 0 & 0 & 3 & 33.3 & 9 \\
\hline More than 10 & 0 & 0 & 5 & 62.5 & 1 & 12.5 & 2 & 25 & 8 \\
\hline
\end{tabular}

Sources, Data obtained from the medical records

Another aspect that was taken into consideration was the intensity of the tobacco habit measured from the number of cigarettes smoked per day. Those individuals in the study who consumed between 20 and 40 cigarettes per day showed percentages distributed in all stages of lung cancer at the time they were diagnosed; and although the highest percentage $(70,2 \%)$ was located in stage IV of the disease, it is striking that $14,9 \%$ was detected in stage II. As for patients who smoked more than 40 cigarettes a day, they emphasize interest given that about $30 \%$ was diagnosed in stage II (Table 2).

Another indicator of intensity in tobacco consumption was smoking time. While $80 \%$ and $87.5 \%$ of the patients who were between 30 and 39 years old and between 40 and 49 years old were smoking stage IV of the disease, respectively, only $47 \%$ of those who covered a range of smoking between 50 and 59 years were located in the most advanced stage of the disease. More striking is the fact that those who smoked for more than 60 years, $80 \%$ were diagnosed in a stage II of the condition, and only $20 \%$ was in stage III (Table 2 ).

Ex-smoker time was another variable of interest in the present work: More than $60 \%$ of those who had quit smoking for a period greater than 10 years were diagnosed in stage II of the disease. Those who had between 5 and 10 years of abstinence, the percentages, were distributed in stages I, II, and IV (Graph 1).

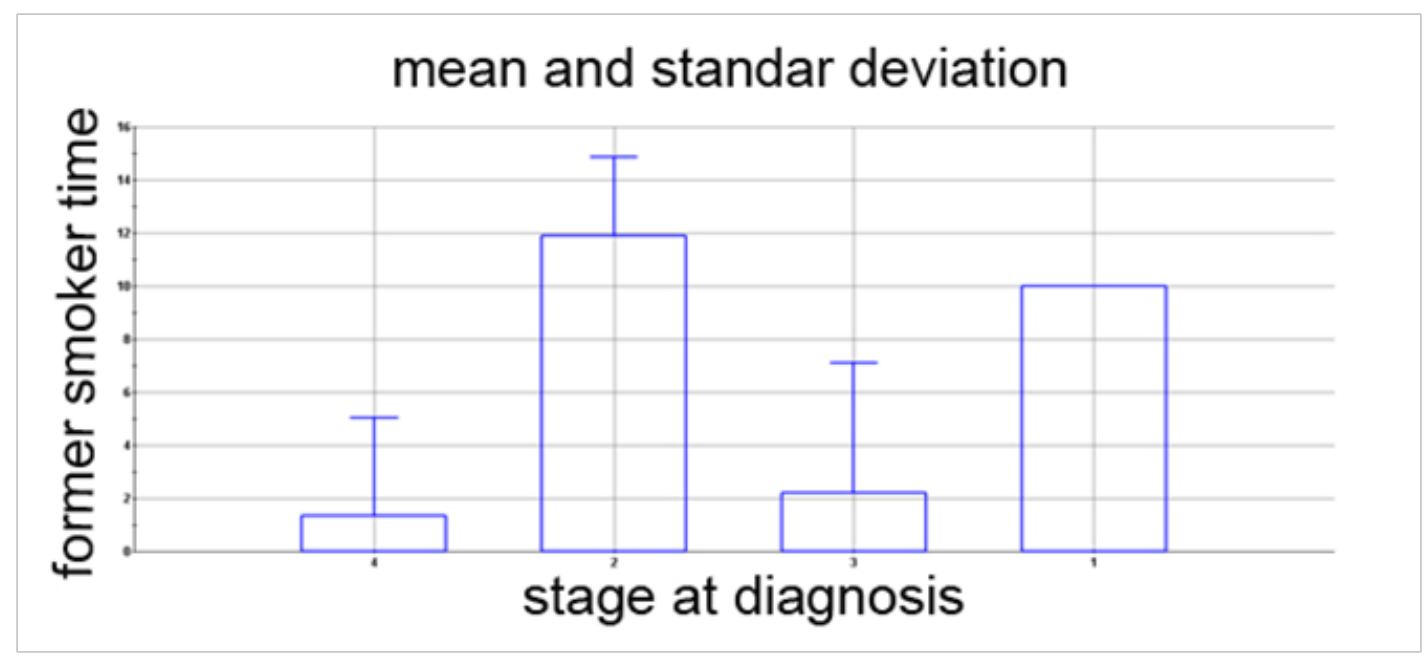

Graph I Relationship between ex-smoker time and stage at diagnosis. 


\section{Discussion}

It is remarkable that, despite the smaller number of women represented in the present study, the percentage of women diagnosed in advanced stages of the disease exceeds that of men. The explanation to this phenomenon does not seem simple. Some studies support the existence of biological and physiological differences between women and men in terms of the degree of susceptibility to exposure of carcinogens derived from tobacco; but the results of these findings are contradictory. ${ }^{7,21,22}$ While some suggest greater vulnerability in women, others say that malignant lung tumors are diagnosed in less advanced stages, evolve with less aggressiveness, and respond better to treatment. ${ }^{23}$ This enters in complete contradiction with the results of this investigation. However, certain explanations are necessary.

The causes that justify gender differences in the incidence of lung cancer include: environmental exposures; genetic differences; molecular abnormalities; hormonal and infection by oncogenic virus. ${ }^{24}$

Among the environmental exposures to which women are more susceptible compared to men are: passive exposure to tobacco smoke; polycyclic aromatic hydrocarbons derived from the combustion of cooking oil in closed environments, radon evolved from concrete walls in small or poorly ventilated rooms. ${ }^{24}$ The family history of cancer, although different from that of the lung, is a risk factor for lung cancer, independent of smoking; and especially affects women; this aspect was not taken into account in the present work, and constitutes a limitation of it. ${ }^{24}$ There are also genes inherited through the germinal route that increase the risk of this type of neoplasm in female patients. They have been mentioned as possible candidates to increase the susceptibility to genes such as: CYP1A1; whose over expression in women is associated with a lower neutralization of carcinogens derived from tobacco at the level of cytochrome P450. Furthermore, mutation of certain genes responsible for repair mechanisms of DNA damage due to the effect of mutagens contained in the cigarette, have been reported with the highest incidence in women under 60 years. ${ }^{24}$ The gastrin-releasing peptide receptor is located on the $\mathrm{X}$ chromosome; and with unusual frequency escapes the inactivation of said chromosome. Gastrin has a strong proliferation-inducing effect in the cells of the bronchial epithelium. A similar effect of estrogens has also been demonstrated at the level of the respiratory mucosa, so women with early menarche, late menopause, obese, nulliparous or with few pregnancies, added to the habit of smoking, are at greater risk of developing a lung neoplasia; interesting association, also revealed in relation to breast cancer, and which supports the evolutionary theory on gender distinctions in terms of life histories. ${ }^{24}$ Asymptomatic infection of the respiratory tract by the human papilloma virus (HPV) in women has been associated with a substantial increase in the risk of lung cancer in nonsmokers; therefore, the added effect of smoking acts synergistically multiplying said risk. ${ }^{24}$ Smoking cessation is much more difficult in female patients than in men; fact clearly illustrated in the results obtained in the present work. ${ }^{25}$ It is pertinent to add, in addition, the higher prevalence of COPD in the participants of this study; factor that conditions not only a higher risk of developing malignant tumors of the lung in those suffering from these conditions, but also supports the paradigm of adaptive oncogenesis, as will be seen later.

It might seem contradictory that while $100 \%$ of patients under 60 years of age were diagnosed in the most advanced stage of lung cancer, about half of those over 75 years of age were diagnosed in stage II. This apparent paradox, however, is explained by the fact that in fact, carcinogenesis at very old ages of life presents its peculiarities, such as lower rates of cell multiplication, lower capacity of tumor angiogenesis, and even lower metastatic capacity of the neoplastic cells. ${ }^{26}$ Among the neoplasm's that share these characteristics is precisely lung cancer. Paired with these observations, there is increasing evidence of the important role played by the inflammatory process during tumor progression; and contrary to what was previously believed, the more intense the inflammatory response "associated with tumors", results in a reliable indication of effective tumor progression, and of course, worse prognosis. ${ }^{27}$ It is unquestionable that the immune response of individuals younger than 60 years is more energetic than those belonging to older ages. However, other alternative answers are also possible; as the lower prevalence of ex-smokers, a lower age of initiation into smoking, genetic predisposition and a more intense dependence on nicotine in patients under 60 years of age. In the present study, none of the 4 patients under 60 was ex-smoker and consumed on average more than 30 cigarettes a day.

Within the subgroup of non-small cell lung cancer, adenocarcinoma represents the most frequent histological variety, an element that coincides with that recorded in the present investigation. ${ }^{28}$ This variety of lung cancer has traits that make it extremely interesting. Although its association with smoking is undeniable, it is also the most prevalent histological subtype in non-smokers, especially in women. In addition, their biological, molecular, and immunological characteristics are markedly different from the squamous cell tumor. Campbell et al examined the exome sequences and gene copy number profile of 1, 144 lung cancers to explore the similarities and differences between adenocarcinoma and squamous cell carcinoma. The comparisons showed that both the mutated genes and the exome sequences are enormously different between the two types of tumors. ${ }^{28}$ There are similarities between squamous cell carcinoma and a subset of bladder tumors; fact that perhaps explains the association between smoking and carcinogenesis of this last organ. The differences suggest that somatic alterations may have a different oncogenic potential, in different cellular contexts. Thus, tumors that originate from cells of similar origin during the development of different tissues may be more similar than those that arise from cells of different origin within the same tissue..$^{28}$ On the other hand, there has been an increase during the last decades in the incidence of lung adenocarcinomas in smokers, surpassing the epidermoid, as a probable consequence of the change in the composition of cigarettes and the inclusion of filters in these. ${ }^{28}$ Unfortunately, this aspect was not present in the present work. Another distinctive feature of adenocarcinoma is that there seems to be an obvious correlation between the number of divisions of stem cells throughout life and the difference in lung cancer risk compared with other histological varieties; so the environmental effect, even the non-tobacco one, could be much lower to induce carcinogenesis. ${ }^{29}$ Perhaps the explanation behind why it was the type of tumor that was diagnosed most in the most advanced stage of the disease lies here.

The age of initiation in smoking as one of the factors that most influence the risk of lung cancer is a point that has strong evidence. The results of this investigation coincide fully with this point of view: there is a directly proportional relationship between the lower age of initiation and the risk of detecting a lung neoplasm in advanced stages. ${ }^{30}$ It represents a sad reality that the average age at which Cubans begin smoking is 12 years. ${ }^{31}$

Why about $30 \%$ of smokers who consumed more than 40 cigarettes a day were diagnosed in stage II of the disease? Result apparently contradictory with what was registere in the literature. ${ }^{30}$ It is necessary 
to emphasize that what is definitely established is the relationship between the number of cigarettes consumed per day and the risk of developing lung cancer; but do not enter this meter of intensity of consumption and the stage of diagnosis of the disease. In other words, the variability in the stage at which the disease is diagnosed does not have to depend on the number of cigarettes consumed. In fact, other factors must intervene that will also be analyzed later. However, other justifications could again be cited for the phenomenon in the present study. For example, $25 \%$ who consumed an average of more than 40 cigarettes a day were also ex-smokers with more than 10 years of habit abandonment. The effect of "memory bias" cannot be ignored either, especially in long-standing ex-smokers; added to the psycho-emotional tendency of those who, after having been longterm smokers, exaggerate the magnitude of consumption during their active stage. ${ }^{32,33}$ Elements that tend to bias the results.

On the other hand, how to explain the fact that among those who smoked for an average of 50-59 years only $47 \%$ was detected in a stage IV; or, much more surprisingly, $80 \%$ of those who smoked for more than 60 years were included in stage II? Again, it is convenient to point out that the perception of these results may be erroneous, since it could assume that the "longer duration of the greatest smoking, or extended, the tumor at the time of detection". This fallacy of logic could in turn be a consequence of the traditional paradigm of the theory of somatic mutation; that conceives to the tumor progression as the sequential accumulation in the time of the necessary mutations so that a tumor fulfils its stages of development. However, in light of current findings, "complete" tumor development is not synonymous with "tumor size"; and apart from the very complex range of intervening factors in the tumor's evolutionary dynamics, the effect of the cellular microenvironment, or ecological context in which the tumor evolution takes place, is already mentioned. That is, more than $50 \%$ of mutations of oncogenic nature accumulate from early stages of life; these mutations remain "contained" within the tissues, since the state of health of the cells that do not carry these mutations is optimal; However, as it ages and is exposed to the harmful action of other environmental mutagens, in this case tobacco smoke, normal cells are losing their degree of adaptive aptitude to the damaged environment, creating a permissible environment for the proliferation of the mutated cells, until then "silenced". ${ }^{34,35}$ In the opinion of the authors of the present study, and starting from the logic established above, initiation at an early age in smoking contributes directly to carcinogenesis, causing an early ecological deterioration of lung tissue, and making mutations, already accumulated during this stage of life, more adaptive, or what is the same, more oncogenic. On the other hand, each individual has genetic, biological, and behavioral peculiarities, which particularize their response to exposure to environmental or endogenous carcinogens (those produced during normal metabolic processes). ${ }^{35}$ In the clinical order, in addition, it is necessary to emphasize the fact that, among the patients who were more than 60 years old, only $20 \%$ consumed more than 40 cigarettes a day; in contrast to the $80 \%$ of those who, during the same period, smoked between 20 and 40 cigarettes a day.

Analogously, it could be explained why former smokers with more than 10 years of abstinence reported the highest percentage among those diagnosed at earlier stages of the disease. It could be suggested that environmentally induced carcinogenesis in general, and smoking in particular, is not limited to the sequential accumulation in time of mutations "essential" for tumor progression; but it is essential the tissue context in which the progression takes place, and the effect of exposure to environmental mutagen. ${ }^{16,17,34,35}$ There is no doubt that all the patients included in this study developed lung cancer; However, a relationship between time without direct exposure to the cigarette and the lower stage at the moment of detecting the tumor is evident, so it would be plausible to assume that although the processes of tumor initiation and progression took place in all individuals, the evolution further neoplasia was "hampered" in patients who quit, perhaps, and according to the theory of adaptive oncogenesis, by persistence, or regeneration of less permissible neoplastic progression ${ }^{16,17}$ cellular microenvironments. Observations parallel to these have been reported by other authors: Mong et al ${ }^{36}$ carried out an impeccable cohort study in this regard; in which $77 \%$ of the patients had a history of previous smoking. ${ }^{36}$ The times of abstinence to tobacco were: less than 1 year, $1-10$ years, $11-20$ years, $21-30$ years, $31-40$ years, $41-50$ years. The average time of abstinence was 18.1 years. In this cohort, the prevalence of lung cancer was higher in those who had stopped smoking one or more decades before developing the tumor. The same authors concluded that smoking exerts an effect on the pathogenesis of lung cancer even years after discontinuing the habit. There is talk of the "bad effect of abandonment", which substantially increases the risk during the first 5 years of abstinence. ${ }^{36}$ In part, it is explained because patients give up the habit when they notice the presence of symptoms such as cough, dyspnea, or hemoptysis; being these initial symptoms of tumor development, often confused with the irritant effect of tobacco smoke. ${ }^{37} 60 \%$ of patients developed the disease after a decade of abstinence. ${ }^{36}$

However, independently of the fact that the risk even in former smokers never reaches the base level of those who have never smoked, several authors emphasize the benefits of smoking abstinence in relation to lung cancer. ${ }^{37}$ Interestingly, individuals carrying the CHRNA5 polymorphism on chromosome $15 \mathrm{q} 25$ have a high risk of developing lung cancer; even 4 years before the rest of the non-carriers of the polymorphism in question. ${ }^{37,38}$ This polymorphism has also been associated with a strong dependence on nicotine, and therefore a more intense consumption, for a long period, and difficulty and delay for the abandonment of the drug. habit Smoking cessation reduces the risk of developing neoplasia, even in these patients, and delays the onset of the disease up to 7 years, when compared with patients who do not carry the polymorphism. ${ }^{37}$ The effect of this is related to the addictive behavior, so that the effort in the detoxification should take this aspect into account. ${ }^{38}$ Also this polymorphism is associated with the risk of COPD; especially the emphysema ; so it seems to have a high prevalence in the population of smokers. Although it is regrettable not to have the means to corroborate it, the sample of the current investigation shares several clinical characteristics with the one cited as the carrier of the polymorphism. ${ }^{37-41}$ On the other hand, Parsons et al demonstrated in their study that although the majority of the patients studied were in an early stage of the tumor when they were diagnosed, continuing to smoke was associated with a significant risk of mortality, recurrence, and develop a second primary tumor. Only $33 \%$ of survival was obtained at 5 years in patients of 65 years who continued smoking against $70 \%$ of those who discontinued the habit. Even in the small-cell, limited-stage variety, $29 \%$ of those who stayed smoking had a 5 -year survival, compared to $69 \%$ of those who left. ${ }^{42}$

However, the most illustrative example of the need for tissue ecological deterioration prior to cancer cell transformation, present in this study, is provided by the undeniable association between the history of COPD and the stage of the lung tumor at the time of diagnosis. Both chronic bronchitis and pulmonary emphysema are characterized by an intense inflammatory response, fibrotic reaction, and the consequent high rates of local cellular proliferation; current 
physiopathological bases that represent the preamble of tumor development. ${ }^{27}$ They are not enough, reiterates the hypothesis of adaptive oncogenesis, oncogenic mutations accumulated over time, but must be coupled, so that they acquire their eventual oncogenic meaning, to an environmental framework (say tissue) conducive to its propagation and fixation at the level of the cell populations that carry them. In the case of women, the special susceptibility of their lung parenchyma to the development of COPD has been made evident, and, together with this, their increased risk of suffering a neoplasm. ${ }^{43}$

\section{Conclusion}

The present study suggests that factors such as female gender, history of COPD, and an age of more initiation early in the HA smoking habit, are predominant clinical features of patients with NSCLC detected in more advanced stages of the disease. At the same time, ex-smoker's time seems to be related to an earlier stage of the disease at the time of diagnosis. The relationship between age, the number of cigarettes smoked per day, or time smoking with the severity of the disease is not clear; it is very probable that other factors intervene, partly explained from the theory of adaptive oncogenesis.

\section{Acknowledgements}

We are grateful to $\mathrm{PhD}$ Joel Fernández-Moreno for suggestions that improved the manuscript. We also thank PhD Alexander ReyesDe La Paz for suggestions and critical review on manuscript, both from “Dr. Luis Díaz Soto” Navy hospital, Havana city. Cuba.

\section{Competing interests}

We declare we have no competing interests.

\section{References}

1. Ferlay J, Soerjomataram I, Dikshit R, et al. Cancer incidence and mortality worldwide: sources, methods and major patterns in GLOBOCAN 2012. Int J Cancer. 2015;136(5):E359-386.

2. Jemal A, Bray F, Center MM, et al. Global cancer statistics. CA Cancer J Clin. 2011;61:69-90.

3. Torre LA, Siegel RL, Ward EM, et al. Global cancer incidence and mortality rates and trends-an update. Cancer Epidemiol Biomarkers Prev. 2016;25(1):16-27.

4. Siegel R, Miller KD, Jemal A. Cancer statistics. CA Cancer J Clin 2017;68(1):7-30.

5. Anuario Estadístico De Cuba 2015: Statistical Yearbook of Health Havana: Ministry of Public Health (CU); 2016. 445 p.

6. Lovly C, Horn L, Pao W. Molecular profiling of lung cancer. My Cancer Genome. 2016.

7. Powell HA, Iyen-Omofoman B, Hubbard RB, et al. The association between smoking quantity and lung cancer in men and women. Chest. 2013;143(1):123-129.

8. Hall F. Reverse translational implications of genome-wide association studies for addiction genetics. In: Preedy V, editor. The neuropathology of drug additions and drug misuse. London: Elsevier; 2016:53-60.

9. Kiyohara C, Ohno Y. Sex differences in lung cancer susceptibility: a review. Gend Med. 2010;7(5):381-401.

10. Cooper WA, Lam DC, O'toole SA, et al. Molecular biology of lung cancer. J Thorac Dis. 2013;5:S479-490.

11. Peto J. That the effects of smoking should be measured in pack-years: misconceptions 4. Br J cancer. 2012;107(3):406-407.
12. Foy M, Spitz MR, Kimmel M, et al. A Smoking-based carcinogenesis model for lung cancer risk prediction. Int $J$ Cancer. 2011;129(8):1907-1913.

13. Merlo LMF, Pepper JW, Reid BJ, et al. Cancer as an evolutionary and ecological process. Nat Rev Cancer. 2006;6(12):924-935.

14. Sprouffske K, Pepper JW, Maley CC. Accurate reconstruction of the temporary order of mutations in neoplastic progression. Cancer Prev Res (Phila). 2011;4(7):1135-1144.

15. Jia P, Pao W, Zhao Z. Patterns and processes of somatic mutations in nine major cancers. BMC Med Genomics. 2014;7:11.

16. Liggett LA, DeGregori J. Changing mutational and adaptive landscapes and the genesis of cancer. Biochim Biophys Acta. 2017;1867(2):84-94.

17. Rozhok A, DeGregori J. Toward an evolutionary model of cancer: Considering the mechanisms that govern the fate of somatic mutations. Proc Natl Acad Sci U S A. 2015;112(29):8914-8921.

18. Tomassetti C, Vogelstein B. Variation in cancer risk among tissues can be explained by the number of stem cell divisions. Science. 2015;347(6217):78-81.

19. Niewoehner DE. Clinical practice: outpatient management of severe COPD. N Engl J Med. 2010;362(15):1407-1416.

20. Miller KD, Siegel RL, Lin CC, et al. Cancer treatment and survivorship statistics, 2016. CA Cancer J Clin. 2016;66(4):271-289.

21. De Matteis S, Consonni D, Pesatori AC, et al. Are women who smoke at higher risk for lung cancer than men who smoke? Am J Epidemiol. 2013;177(7):601-612.

22. Risch HA, Howe GR, Jain M, et al. Are female smokers at higher risk for lung cancer than male smokers? A case-control analysis by histologic type. Am J Epidemiol. 1993;138(5):281-293.

23. Malvezzi M, Bertuccio P, Rosso T, et al. European cancer mortality predictions for the year 2015: Does lung cancer have the highest death rate in EU women? Ann Oncol. 2015;26(4):779-786.

24. North CM, Christiani DC. Women and Lung Cancer: What's New? Sem in Thorac Cardiovasc Surg. 2014;25(2):1-13.

25. Pirie K, Peto R, Reeves GK, et al. The 21st century hazards of smoking and benefits of stopping: a prospective study of one million women in the UK. Lancet. 2013;381(9861):133-141.

26. Falandry C, Bonnefoy M, Freyes G, et al. Biology of cancer and aging: A complex association with cellular senescence. Jour Clin Oncol. 2014;32(24):2604-2610.

27. Brücher B, Jamall IS. Epistemology of the origin of cancer: a new paradigm. BMC Cancer. 2014;14:331

28. Campbell JD, Alexandrov A, Kim J, et al. Cancer genome atlas research network. distinct patterns of somatic genome alterations in lung adenocarcinom as and squamous cell carcinomas. Nat Genet. 2016;48(6):607-616

29. Tomasetti C, Marchionnic L, Nowak MA, et al. Only three driver mutations are required for the development of lung and colorectal cancers. Proc Natl Acad Sci U S A. 2016;112(1):118-123.

30. Jha P, Ramasundarahettige C, Landsman V, et al. 21st-century hazards of smoking and benefits of cessation in the United States. $N$ Engl J Med. 2013;368(4):341-350.

31. Suárez Lugo N. Market and consumption of cigarettes in Cuba and the decision between tobacco or health. Cuban Revista de Salud Pública. 2014;40(3):331-344.

32. Kang MI, Ikeda S. Time discounting and smoking behavior: evidence from a panel survey. Health Econ. 2014;23(12):1099-1050. 
33. Lin W, Sloan F. Risk perceptions and smoking decisions of adult Chinese men. J Health Econ. 2015;39:60-73.

34. Scott J, Marusyk A. Somatic clonal evolution: A selection-centric perspective. Biochim Biophys Acta. 2017;1867(2):139-150.

35. Gatenby RA, Brown J. Mutations, evolution and the central role of a self-defined fitness function in the initiation and progression of cancer. Biochim Biophys Acta. 2017;1867(2):162-166.

36. Mong C, Garon EB, Fuller C, et al. High prevalence of lung cancer in a surgical cohort of lung cancer patients a decade after smoking cessation. J Cardiothorac Surg. 2011;6(19):1-7.

37. Hall FS. Genetic risk for lung cancer and the benefits of quitting smoking. EBioMedicine. 2016;11:19-20.

38. Chen LS, Baker T, Hung RJ, et al. Genetic risk can be decreased: quitting smoking decreases and delays lung cancer for smokers with high and low CHRNA5 risk genotypes - a meta-analysis. EBioMedicine. 2016;11:219-226.
39. Bergen AW, Javitz HS, Krasnow R. Nicotinic acetylcholine receptor variation and response to smoking cessation therapies. Pharmacogenet Genomics. 2013;23(2):94-103.

40. Bloom AJ, Hartz SM, Baker TB., et al. Beyond cigarettes per day. A genome-wide association study of the biomarker carbon monoxide. Ann Am Thorac Soc. 2014;11(7):1003-1010.

41. Chen LS, Hung RJ , Baker T, et al. CHRNA5 risk variant predicted delayed smoking cessation and earlier lung cancer diagnosis-a metaanalysis. J Natl Cancer Inst. 2015;107(5).

42. Parsons A, Daley A, Begh B, et al. Influence of smoking cessation after diagnosis of early stage lung cancer on prognosis: systematic review of observational studies with meta-analysis. BJM. 2010;340:5569.

43. Young RP, Hopkins RJ, Whittington CF, et al. Individual and cumulative effects of GWAS susceptibility loci in lung cancer: associations after sub-phenotyping for COPD. PLoS One. 2011;6(2):e16476. 